\section{Scalar Scoring in Thalassemia Genotype: As a New Overview}

Received: September 26, 2017; Accepted: October 18, 2017; Published: October 26, 2017

\section{Introduction}

One of the most complex subjects of Hematology is thalassemia and hemoglobinopathy genotype interpretation and phenotype determination as well as their clinical symptoms and electrophoresis. Therefore, establishing a logical digital relationship between the above-mentioned items can contribute to the understating of this subject.

In this opinion article, we present a new digital scoring method for genotype and predicting phenotype and some $\mathrm{CBC}$ indexes based on estimated genotype score. This method in Hematology might facilitate the complexity of medical patients, but it may not be possible to justify all existing details in these patients.

\section{Material and Methods}

Focusing on down table: For example, a 6 -year-old patient with $\beta^{A} / \beta^{+}$genotype is a minor $\beta$ thalassemia case and he has $\beta$ score $=4$ and $\alpha$ score $=6$ and total score $=10$ in our scoring. In this method, we attribute his minimum $\mathrm{Hb}$ as a sum of $\alpha$ score and $\beta$ score equal 10 and his minimum $M C V=76$. Also, in this patient because of $\beta$ thalassemia minor, genotype is asymptomatic with predictable $\mathrm{Hb}$ electrophoresis.

The thalassemia has Mendelian inheritance and it has inherited autosomal recessive; therefore, people with $50 \%$ healthy alleles have thalassemia trait and they are not patients, but the people with $100 \%$ involved alleles of $\alpha$ or $\beta$ genes have thalassemia disease and they are patients. Thalassemic patients have organomegaly, anemia, and ineffective erythropoiesis with objective and subjective symptoms (fatigue, pallor, jaundice) and abnormal $\mathrm{Hb}$ electrophoresis and $\mathrm{CBC}$, but thalassemia trait persons just have abnormal $\mathrm{Hb}$ electrophoresis and $\mathrm{CBC}$ without clinical symptoms (Figure 1).

In this method, we attributed $\beta$ chain score equal 3.

$\beta^{A}, \beta^{+}, \beta^{0}$ and 6 compound genotypes derive from this three conditions. Every healthy $\beta$ chain gets the score +3 : $\mathrm{HbA}$ has $\alpha_{2} \beta_{2}$ combination; therefore, a healthy person with two healthy $\beta$ chains in hemoglobin $A$ is shown as $\beta^{A} / \beta^{A}$ has $\beta$ score $=+6$, So: (Figure 2a-c).

Hemoglobin Lepore is a chimeric protein, which is resulted from a fusion gene (adherence of $\beta$ gene to $\Delta$ ). Therefore: (Figure 3 ).

Based on the above description, Lepore trait has $\beta$ score $=+4$; phenotypes.

\section{Babak Abdolkarimi ${ }^{1 *}$, Javad Dehbozorgian² and Shahrzad Razmjooee ${ }^{2}$}

\section{*Corresponding author: Abdolkarimi B \\ b.abdolkarimi@yahoo.com}

Assistant Professor of Pediatric Hematology and Oncology Department, Lorestan University of Medical Sciences, Iran.

Tel: +9183605274

Citation: Abdolkarimi B, Dehbozorgian J, Razmjooee S (2017) Scalar Scoring in Thalassemia Genotype: As a New Overview. Appl Sci Res Rev Vol. 4 No.2:9

therefore, the patient has minor thalassemia and Lepore homozygous patient has $\beta$ score $=+2$ and the patient has intermediate thalassemia. With the same method composition of $B^{\text {lepore }}$ with other $\beta$ gene variations such as $\beta^{\mathrm{E}}, \beta^{\mathrm{C}}, \beta^{\mathrm{S}}, \beta^{D}$ produced other $\beta$ thalassemia and hemoglobinopathies genotypes and

Exception 1: $\beta^{\text {lepore }} / \beta^{\text {lepore }}$ (homozygote Lepore or Lepore disease) is one of exceptions, which has maximum $20-25 \%$ Lepore $\mathrm{Hb}$ with score $=2$. Therefore, he is an intermediate $\beta$ thalassemia phenotype or disease. This patient has $75-80 \% \mathrm{HbF}$. People with Lepore heterozygote genotype such as $\beta^{\text {lepore }} \beta^{A}$ have score $=4$; therefore, they are $\beta$ variant thalassemia trait and have no disease. In these cases, $\mathrm{HbA} 1$ and $\mathrm{HbA} 2$ do not exist and remaining $\mathrm{Hb}$ chains are $75-80 \% \delta$ chains that present with $\mathrm{HbF}$.

Note: Second interesting genotype in $\beta$ thalassemia is co-deletion of $\beta$ gene and ${ }^{\Delta}$. One of this genotypes is $(\Delta \beta)^{+} /(\Delta \beta)^{+}$. This person has $\beta$ score $=+2$ and he is $\beta$ thalassemia intermediate with low $\mathrm{HbA} 2$, $\operatorname{HbA} 1 .(\Delta \beta)^{+} / A$ is heterozygous with score $=+4$; therefore, he is a case of $\beta$ thalassemia trait or minor $\beta$ thalassemia with low $\mathrm{HbA} 2$. 


\begin{tabular}{|c|c|c|c|c|}
\hline THALASSEMIA & $\begin{array}{l}\text { GLOBIN } \\
\text { GENOTYPE }\end{array}$ & FEATURES & EXPRESSION & $\begin{array}{l}\text { HEMOGLOBIN } \\
\text { ANALYSIS }\end{array}$ \\
\hline \multicolumn{5}{|l|}{$\alpha$-THALASSEMIA } \\
\hline 1 gene deletion & $-\alpha / \alpha, \alpha$ & Normal & Normal & Newborn: Bart's 1-2\% \\
\hline 2 gene deletion trait & $-, \alpha /-, \alpha-,-/ \alpha, \alpha$ & $\begin{array}{l}\text { Microcytosis, mild } \\
\text { hypochromasia }\end{array}$ & Normal, mild anemia & Newborn: Bart's: $5-10 \%$ \\
\hline $\begin{array}{l}3 \text { gene deletion } \\
\text { hemoglobin } \mathrm{H}\end{array}$ & $-,-1, \alpha$ & Microcytosis, hypochromic & $\begin{array}{l}\text { Mild anemia, transfusions not } \\
\text { required }\end{array}$ & Newborn: Bart's: $20-30 \%$ \\
\hline $\begin{array}{l}2 \text { gene deletion + } \\
\text { Constant Spring }\end{array}$ & $\begin{array}{l}-,-/ \alpha, \alpha \text { Constant } \\
\text { Spring }\end{array}$ & Microcytosis, hypochromic & $\begin{array}{l}\text { Moderate to severe anemia, } \\
\text { transfusion, splenectomy. }\end{array}$ & $\begin{array}{l}2-3 \% \text { Constant Spring, } \\
10-15 \% \text { hemoglobin } \mathrm{H}\end{array}$ \\
\hline 4 gene deletion &,,,---- & Anisocytosis, poikilocytosis & Hydrops fetalis & $\begin{array}{l}\text { Newborn: } 89-90 \% \text { Bart's } \\
\text { with Gower } 1 \text { and } 2 \text { and } \\
\text { Portland }\end{array}$ \\
\hline Nondeletional & $\alpha, \alpha / \alpha, \alpha$ variant & Microcytosis, mild anemia & Normal & $1-2 \%$ variant hemoglobin \\
\hline \multicolumn{5}{|l|}{$\beta$-THALASSEMIA } \\
\hline $\begin{array}{l}\beta^{0} \text { or } \beta^{+} \\
\text {heterozygote: trait }\end{array}$ & $\beta^{\circ} / A, \beta+/ A$ & Variable microcytosis & Normal & $\begin{array}{l}\text { Elevated } A_{2} \text {, variable } \\
\text { elevation of } F\end{array}$ \\
\hline$\beta^{0}$-Thalassemia & $\begin{array}{l}\beta^{0} / \beta^{0}, \beta^{+} / \beta^{0} \\
E / \beta^{0}\end{array}$ & $\begin{array}{l}\text { Microcytosis, nucleated } \\
\text { RCB }\end{array}$ & Transfusion dependent & $\begin{array}{l}\mathrm{F} 98 \% \text { and } \mathrm{A}_{2} 2 \% \\
\text { E } 30-40 \%\end{array}$ \\
\hline $\begin{array}{l}\beta^{+} \text {-Thalassemia } \\
\text { severe }\end{array}$ & $\beta^{+} / \beta^{+}$ & $\begin{array}{l}\text { Microcytosis nucleated } \\
\text { RBC }\end{array}$ & $\begin{array}{l}\text { Transfusion dependent/thalassemia } \\
\text { intermedia }\end{array}$ & $\begin{array}{l}F 70-95 \%, A_{2} 2 \% \text {, trace } \\
\text { A }\end{array}$ \\
\hline Silent & $\beta+/ A$ & Microcytosis & Normal with only microcytosis & $A_{2} 3.3-3.5 \%$ \\
\hline$\beta^{+} / \beta^{+}$ & $\begin{array}{l}\text { Hypochromic, } \\
\text { microcytosis }\end{array}$ & Mild to moderate anemia & $\mathrm{A}_{2} 2-5 \%, \mathrm{~F} 10-30 \%$ & \\
\hline Dominant (rare) & $B^{\circ} / A$ & $\begin{array}{l}\text { Microcytosis, abnormal } \\
\text { RBCs }\end{array}$ & $\begin{array}{l}\text { Moderately severe anemia, } \\
\text { splenomegaly }\end{array}$ & Elevated $\mathrm{F}$ and $\mathrm{A}_{2}$ \\
\hline$\delta$-Thalassemia & A/A & Normal & Normal & $\mathrm{A}_{2}$ absent \\
\hline$(\delta \beta)^{0}$-Thalassemia & $(\delta \beta)^{0} / A$ & Hypochromic & Mild anemia & F $5-20 \%$ \\
\hline $\begin{array}{l}(\delta \beta)^{+}-\text {Thalassemia } \\
\text { Lepore }\end{array}$ & $\beta$ Lepore /A & Microcytosis & Mild anemia & Lepore $8-20 \%$ \\
\hline Lepore & $\beta$ Lepore $/ \beta$ Lepore & Microcytic, hypochromic & Thalassemia intermedia & F $80 \%$, Lepore $20 \%$ \\
\hline
\end{tabular}

Figure 1 Genotype and phenotype of $\beta$ thalassemia and clinical features.
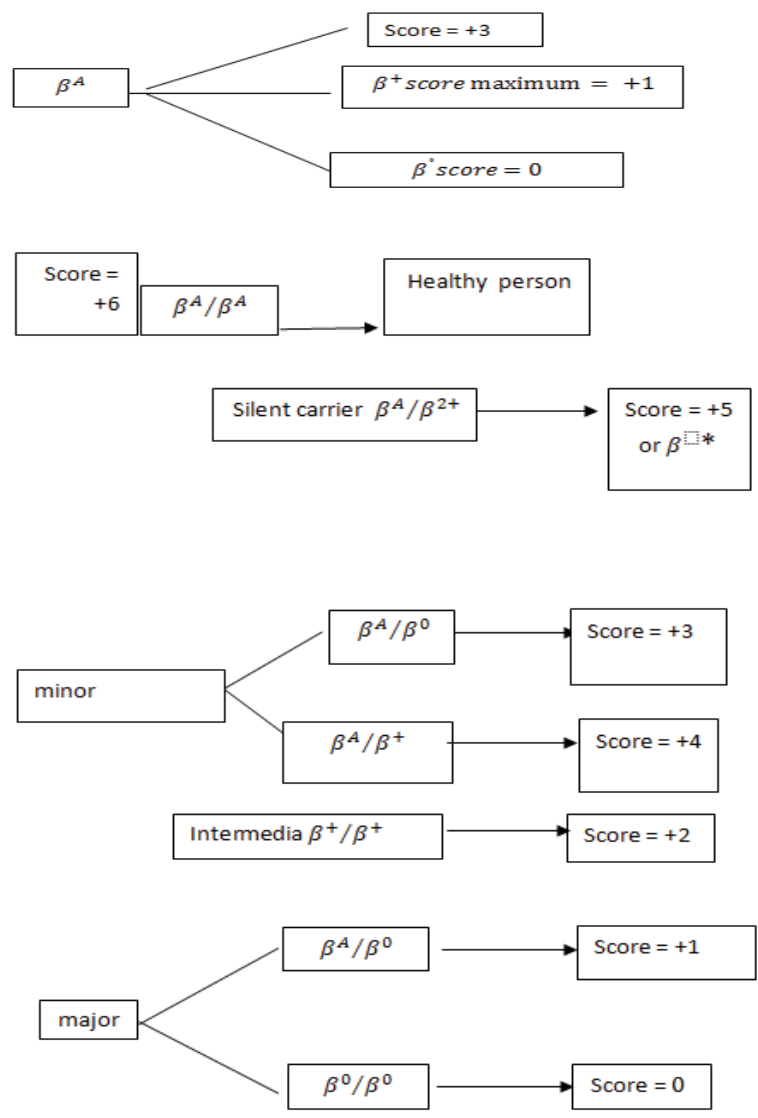

Figure 2 (a-c) Healthy person with two healthy $\beta$ chain in hemoglobin $A$ shown as $\beta^{\wedge} A / \beta^{\wedge} A$ has $\beta^{\wedge}$ score $=+6$. 


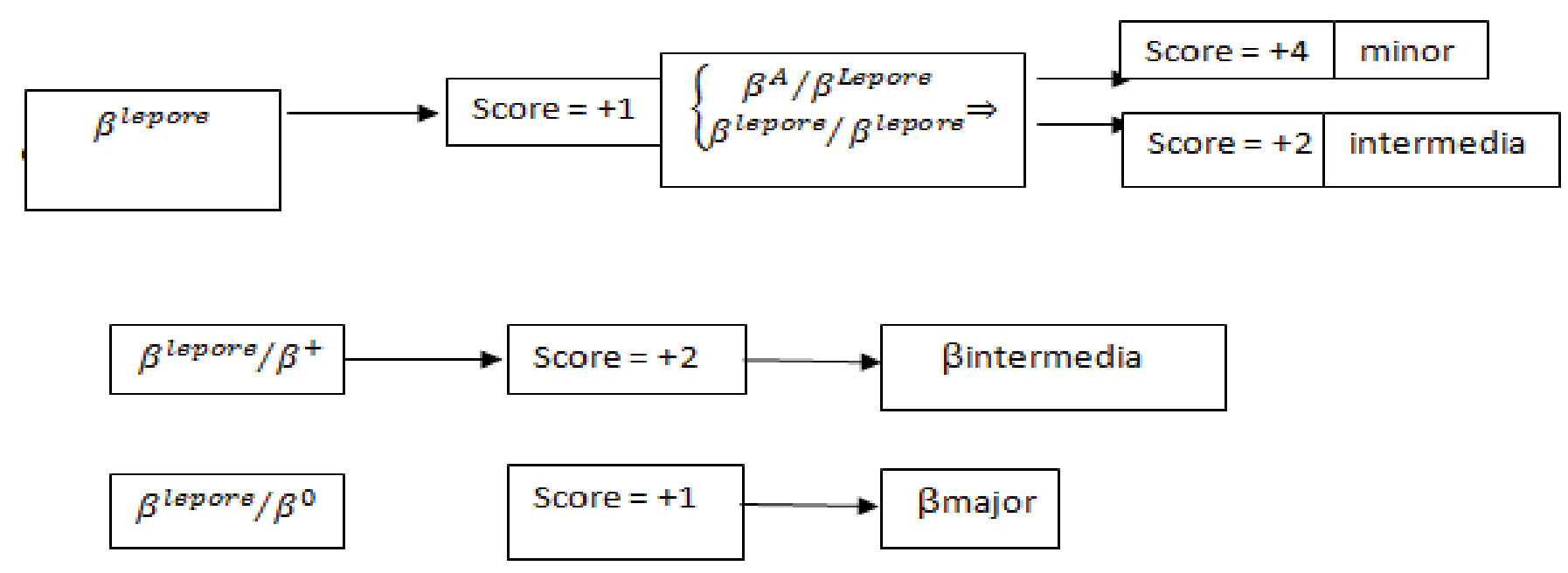

Figure 3 Scoring of Lepore trait.

\begin{tabular}{|l|l|l|l|l|l|}
\hline Gen/genotype & $\boldsymbol{\beta}^{\mathrm{A}}$ & $\boldsymbol{\beta}^{2+}$ & $\boldsymbol{\beta}^{+}$ & $\boldsymbol{\beta}^{\mathrm{O}}$ & $\boldsymbol{\beta}^{\text {lepore }}$ \\
\hline $\boldsymbol{\beta}^{\mathbf{A}}$ & $\beta^{\mathrm{A}} \beta^{\mathrm{A}}$ & $\beta^{\mathrm{A}} \beta^{2+}$ & $\beta^{\mathrm{A}} \beta^{+}$ & $\beta^{\mathrm{A}}$ & $\beta^{\mathrm{A}} \beta^{\text {lepore }}$ \\
\hline $\boldsymbol{\beta}^{2+}$ & $\beta^{2+} \beta^{\mathrm{A}}$ & $\beta^{2+} \beta^{2+}$ & $\beta^{2+} \beta^{+}$ & $\beta^{2+}$ & $\beta^{2+} \beta^{\text {lepore }}$ \\
\hline $\boldsymbol{\beta}^{+}$ & $\beta^{+} \beta^{\mathrm{A}}$ & $\beta^{+} \beta^{2+}$ & $\beta^{+} \beta^{+}$ & $\beta^{+}$ & $\beta^{+} \beta^{\text {lepore }}$ \\
\hline $\boldsymbol{\beta}^{\mathrm{O}}$ & $\beta^{\mathrm{O}} \beta^{\mathrm{A}}$ & $\beta^{\mathrm{O}} \beta^{2+}$ & $\beta^{\mathrm{O}} \beta^{+}$ & $\beta^{\mathrm{O}}$ & $\beta^{\mathrm{O}} \beta^{\text {lepore }}$ \\
\hline $\boldsymbol{\beta}^{\text {lepore }}$ & $\beta^{\text {lepore }} \beta^{\mathrm{A}}$ & $\beta^{\text {lepore }} \beta^{2+}$ & $\beta^{\text {lepore }} \beta^{+}$ & $\beta^{\text {lepore }} \beta^{\mathrm{O}}$ & $\beta^{\text {lepore }} \beta^{\text {lepore }}$ \\
\hline
\end{tabular}

Figure 4 Matrix of possible $\beta$ thalassemia genotype.

\begin{tabular}{|l|l|l|l|l|l|}
\hline Gen/genotype & $\boldsymbol{\beta}^{\mathbf{A}}$ & $\boldsymbol{\beta}^{\mathbf{2 +}}$ & $\boldsymbol{\beta}^{+}$ & $\boldsymbol{\beta}^{\mathbf{0}}$ & $\boldsymbol{\beta}^{\text {lepore }}$ \\
\hline $\boldsymbol{\beta}^{\mathbf{A}}$ & 6 & 5 & 4 & 3 & 4 \\
\hline $\boldsymbol{\beta}^{\mathbf{2 +}}$ & 5 & 4 & 3 & 2 & 3 \\
\hline $\boldsymbol{\beta}^{+}$ & 4 & 3 & 2 & 1 & 2 \\
\hline $\boldsymbol{\beta}^{\text {o }}$ & 3 & 2 & 1 & 0 & 1 \\
\hline $\boldsymbol{\beta}^{\text {lepore }}$ & 4 & 3 & 2 & 1 & $\mathbf{2}$ \\
\hline
\end{tabular}

Figure 5 Matrix of score of $\beta$ thalassemia genotype.

\begin{tabular}{|c|c|c|c|c|c|}
\hline Gen/genotype & $\boldsymbol{\beta}^{\mathbf{A}}$ & $\beta^{2+}$ & $\boldsymbol{\beta}^{+}$ & $\beta^{\circ}$ & $\beta^{\text {lepore }}$ \\
\hline$\beta^{A}$ & & Silent Carrier $\beta$ & $\operatorname{minor} \beta$ & $\operatorname{minor} \beta$ & $\operatorname{minor} \beta$ \\
\hline$\beta^{2+}$ & Silent Carrier $\beta$ & $\min o r \beta$ & $\operatorname{minor} \beta$ & $\beta$ intermediate & $\min o r \beta$ \\
\hline $\boldsymbol{\beta}^{+}$ & $\min o r \beta$ & $\min o r \beta$ & $\beta$ intermediate & $\beta$ major & $\beta$ intermediate \\
\hline$\beta^{\circ}$ & $\operatorname{minor} \beta$ & $\beta$ intermediate & $\beta$ major & $\beta$ major & $\beta$ major \\
\hline$\beta^{\text {lepore }}$ & $\operatorname{minor} \beta$ & $\operatorname{minor} \beta$ & $\beta$ intermediate & $\beta$ major & $\beta$ intermediate \\
\hline
\end{tabular}

Figure 6 Matrix of possible $\beta$ thalassemia phenotype.

Genotype and phenotype multiplication table: In summary, if we assess all probable $\beta$ genotypes. Figure 1 shows possible we put all $\beta$ genes situations in horizontal and vertical column, genotypes with $\beta$ gene variations, Figure 3 shows possible 


$$
\begin{aligned}
& \alpha 1 \text { score }=+1 \\
& \alpha 2 \text { score }=+2
\end{aligned}
$$

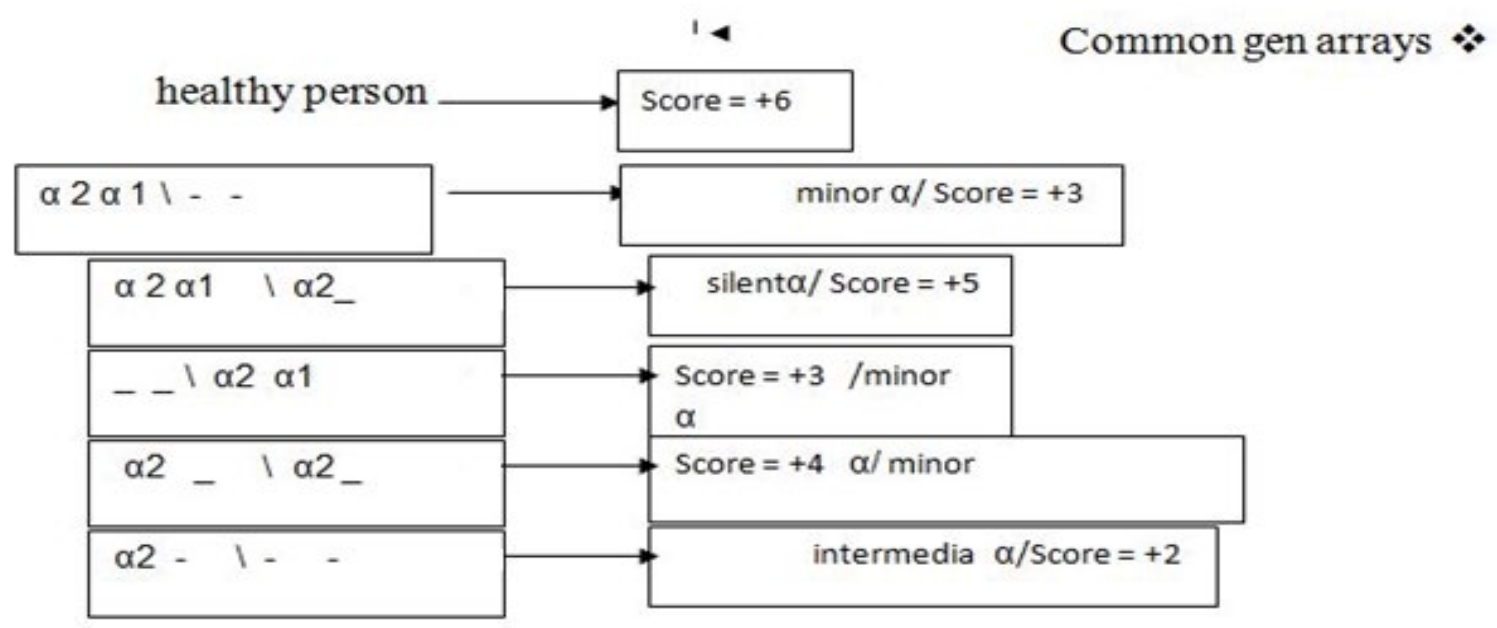

\section{Every constant spring mutation in the $\alpha$ gene} gene to zero.

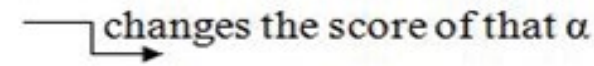

Figure $7 \alpha$ genes placement and the score in $\alpha$ thalassemia.

genotypes score in our scalar scoring, and Figure 4 shows possible clinical situations compatible with genotype and score. For example, patient with $\beta^{+} / \beta^{+}$in matrix home $4 \times 4$ has $\beta$ thalassemia intermediate genotype and $\beta$ score $=+2$ in our scoring (Figures 4-6).

$\alpha$ genes placement and the score in $\alpha$ thalassemia: There are two types of $\alpha$ genes: $\alpha 1$ and $\alpha 2$ genes and they are not equal regarding their ability for producing $\alpha$ chains. Therefore, they have 2 different scalar scores in our scoring system. In healthy persons, $\alpha$ genes composition is $\alpha 2 \alpha 1 \backslash \alpha 2 \alpha 1$. If we attribute $\alpha 1$ score $=+1$ and $\alpha 2$ score $=+2$ in scoring, they have 2 different genotypes with $\alpha$ genes in $\alpha$ thalassemia setting: (Figure 7).

This method is extended about $\alpha$ variants hemoglobinopathies such as Constant Spring Hemoglobin and other deletional and non-deletional genotypes:

$\alpha$ CS score has value 0 in our scoring system, for example: $\alpha 2 \ldots$ $\alpha$ CS_ has score $=+2$, therefore, he is intermediate $\alpha$ thalassemia.

Estimation of complex genotype clinical manifestations: In complex genotype such as $\alpha$ thalassemia intermediate with $\beta$

\section{References}

1 Kliegman R, Stanton B, St. Geme J, Schor N, Behrman RE (2011) 19th edition, Nelson Textbook of Pediatrics. Elsevier Saunders, Philadelphia, USA.

2 Al-Allawi NAS, Jalal SD, Mohammad AM, Omer SQ, Markous RSD (2014) $\beta$-Thalassemia Intermedia in Northern Iraq. Hindawi Publishing Corporation, Egypt. thalassemia minor scor $\alpha=+2$ and $\beta$ thalassemia minor score $=+3$ or +4 and $\alpha / \beta$ ratio $=2 / 3$ or 4 . In pure genotype such as $\alpha$ thalassemia intermediate with $\beta$ normal scor $\alpha=+2$ and $\beta$ score $=+6$ and $\alpha / \beta$ ratio $=2 / 6$. In comparison of two patients, $\alpha / \beta$ ratio nearer to 1 (normal genotype score in healthy person) clinical manifestations would be better.

\section{Conclusion}

Our new and simplified method in interpreting thalassemia genotype, phenotype, and clinical symptoms well based on the scoring method. Therefore, it is an effective applied method for learning, interpreting, and simplifying complicated concepts of thalassemia syndromes. We think that using simple language is most important factor for effectiveness of this course and this method similar case based bedside teaching provides active learning in real context observes students skills, increases learners' motivation and professional thinking, integrates clinical, communication, problem solving, decision making and ethical skills [1-5].

3 Rahim F (2008) Genotyping of Thalassemia in Microcytic Hypochromic Anemia Patients from Southwest Region of Iran. Pak J Med Sci 24: 23-28.

4 George E, Ann TJ (2011) Genotype-Phenotype Diversity of BetaThalassemia in Malaysia: Treatment Options and Emerging Therapies. Med J Malaysia 65: 256-260.

5 Shahriari M (2014) Case Based Teaching at the Bed Side Versus in Classroom for Undergraduates and Residents of Pediatrics. J Adv Med Educ Prof 2: 135-136. 